\title{
Using MATLAB to Get the Best Performance with Different Type Median Filter on the Resolution Picture
}

\author{
${ }^{1}$ Dr. Yahya Ali ALhussieny
}

\begin{abstract}
For preserving edges and removing impulsive noise, the median filter is well known for this purpose. The state of the art of median filter design is reviewed. Compared in terms of performance, several techniques are discussed and implementation feasibility in different type median filters. Resolution of chosen pixel is clearly appeared for median filter using MATLAB has been implemented and designed. Characteristics and performance of the filter has been illustrated in the tables in the results part of the paper.
\end{abstract}

Keywords---Pixel, Digital image processing, Median filter, Neighborhood, Mean filter (average filter), Linear \& non-linear filter, Impulse noise (salt \& pepper noise), Image smoothing

\section{INTRODUCTION}

In the analysis of image, one of the most main image handling steps is being the filtering. In image processing and computer view, filtering is the most basic processes that, for the image filtering, numerous filtering techniques have been invented.

A small adjacency of the same position, is the value of input image, has been a specific position to filter image. The near pixels will be likely to have identical values, when we assume that images are changed slowly over space. but at the regions that contain image details and edges (e.g. end of lines, corners etc.), this result is fail (it means not constant) [2].

Both types of non-linear and linear filters are used in the processing and image enhancement confused. The non-linear filters are more efficient at getting rid of the noise have been proven by researcher.

For processing image confusing, one of these nonlinear filters, is median filter based on fuzzy filter. This filter is characterized by preserving the edges of the images, some research has proved, after the improvement in contrast to some other filters that found to be efficient at getting rid of salt and pepper noise, and also damage the edges.

Based on "degrees of truth" rather than frequent "false or true" (1 or 0) Boolean logic is fuzzy logic method on which the new computer is based. Dr. Lotfi Zadeh is first advanced the idea of fuzzy from University of California at Berkeley in the 1960's [1]. The Centre Weighted Median filter (CWM) is proposed by KO and Lee, so that they applied the output of the filter will take the median of the extended set and the center inside the sliding window or the weight equalization to the origin pixel but downstairs in noise reduction [2]. J.-H. W. A. H.-C. CHIU are proposed to extend the median operation to 2_D such as square mask, as nonlinear technique, in image processing [3].
Although many of filters such as pixel -wise MAD (PWMAD), adaptive center weighted median (ACWM), signal depends order mean (SD-ROM) filter and the mediator multistate (MSM) filter are proposed with the reagent batch to improve the median filter and they are usually a good performance but are higher than $30 \%$ of the noise level, they tend to keep a lot of noise boost or to remove many of the features of the pictures [4].

For removing batch noise from those filters, based on strong statistics, is appeared in [5].

Fuzzy filters, usually, can be distributed into two broad type i.e. one, in which the fuzzy filter is depend on the fuzzification of one or more classical ways of filtering and two, in which the fuzzy filter is completely depend on fuzzy techniques. Many fuzzy filters can be distributed fuzzy switching filters; fuzzy median filter (FMF), Adaptive Weighted Mean Filter (AWMF), etc. have furthermore been discussed for the reducing of impulse noise [2].

\section{FILTERING}

\section{A. Processing of filter}

One of the most main image handling steps, in the analysis of image, is the filtering. For the image filtering, numerous filtering techniques have been invented. The most basic processes, in computer view and image processing, is the filtering. At a specific position, in a small adjacency of the same position, the filtered image is a function of the values of the input image. The near pixels will be likely to have identical values when we assume that images are changed slowly over space. But at the regions that contain edges and image details (e.g. end of lines, corners, lines etc.) this result is not constant (it mean fail)[6].

The Centre Weighted Median filter (CWM) is proposed by the Ko and Lee to trading off detail protection against noise minimizing. they applied the weight equalization to the center inside the sliding window or the origin pixel and the output of the filter will take the median of the extended set .So, the doing of the (CWM) filter with a larger center of weight, in detail protection but downstairs in noise reduction, is ascendant to the one with a smaller center weight when it is observed [2]. 


\section{B. The effectively remove impulse noise}

Ideally, the filtering must be applied just to the noise free pixels must be preserved unchanged and the pixels was corrupted, for keeping image details.

The (CWM) filter was conditioning the pixel exchanging to their ranks in the organizer set without specifying any weights when an effective way to optimize the performance [2].

For keeping image details and removing impulsive noise, Median filtering (MF) is one way that used. It is also the groundwork upon which most advanced image filters like rank-order processing, unsharp masking [3] . So, the MF such as in one dimensional, it's processes is realized when the data passing through the filter it take median value of the data as the output so that it used keeping image details and to remove impulsive noise. While in this paper, too the 2_D(two dimensional) MF is used.

\section{REPRESENTATION THE 2D MEDIAN FILTER}

In any form of (circular disk, central uniform, a square shape, a cross shape or rectangular shape)can be representation the two dimension of median filter.

According to intensity of the pixel, by the median value of all pixels inside the window, can be replace the pixel of the center.

For example, in figure (3_1) below, we will replace the pixel (150) by the median pixel (124). Here we use median filter $3 \mathrm{X} 3$ neighborhood

\begin{tabular}{|l|l|l|l|l|}
\hline 123 & 125 & 126 & 130 & 140 \\
\hline 122 & 124 & 126 & 127 & 135 \\
\hline 118 & 120 & 150 & 124 & 134 \\
\hline 119 & 115 & 119 & 123 & 133 \\
\hline 111 & 116 & 110 & 120 & 130 \\
\hline
\end{tabular}

Neighborhood value for (150) is:

$(115,119,120,123, \mathbf{1 2 4}, 125,126,127,150)$ So the median value is (124).

We can rerun the median filter on the image while there are no more changes. So the median filter does like a maximum expectation encore [6].

For example, in figure (2_6) below: its original image of $3 \mathrm{X} 3$ size.

\begin{tabular}{|l|l|l|}
\hline 91 & 50 & 90 \\
\hline 77 & 68 & 95 \\
\hline 115 & 151 & 210 \\
\hline
\end{tabular}

Fig.3-2 image of $3 \times 3$ size

To use median filter on it, we will use median filter of $3 \mathrm{X} 3$ neighborhood

We will work ascending order for the values of the pixels in this image:

$55,68,77,90,91,95,115,151,210$

So the Median value $\mathbf{= 9 1}$.
IV. THE ALGORITHM TO IMPROVE IMAGE BY USING MEDIAN FILTER

\section{A. calculation equation}

For apply the algorithm to improve image by using median filter follow next steps:

1) Read the picture with the bad lighting.

2) Do ascending order for each pixel in image.

3) Extract the median value of these pixels ranked in descending order.

4) The standard deviation using the median of the previous step, the extracted value is calculated according to the equation below [7]:

$$
S d=\sqrt{\frac{1}{m * n-1} \sum(i(m, n)-\text { median })^{2}}
$$

5) Find a formula to improve the contrast of each pixel of the image pixels and then display the resulting image after optimization, as in the equation below:

$\operatorname{median}(m, n)=K 1\left[\frac{\text { median }}{s d}\right][i(m, n)-$ median $]+K 2 *$

median 3-2

where $\mathrm{K} 1=0.9, \mathrm{~K} 2=1.0$. Fig.3.1 shows this result.

\section{B. To determine the improvement of the filter}

After the application of algorithms to improve the contrast can be indicate the quality of images obtained using as a measure of RMSe process compared between the original image with the results obtained from the) root mean square error (application algorithm each separately, equation below [7].

$$
R M S e=\sqrt{\frac{1}{m * n} \sum_{r=0}^{m-1} \sum_{c=0}^{n-1}\left[I^{\prime}(r, c)-I(r, c)\right]^{2}}
$$

Fig.3.1 2D median filter

where, $\left(m^{*} n\right)$ represents the total size of the image, I represent the original image and I' represent the resulting image after the application. 


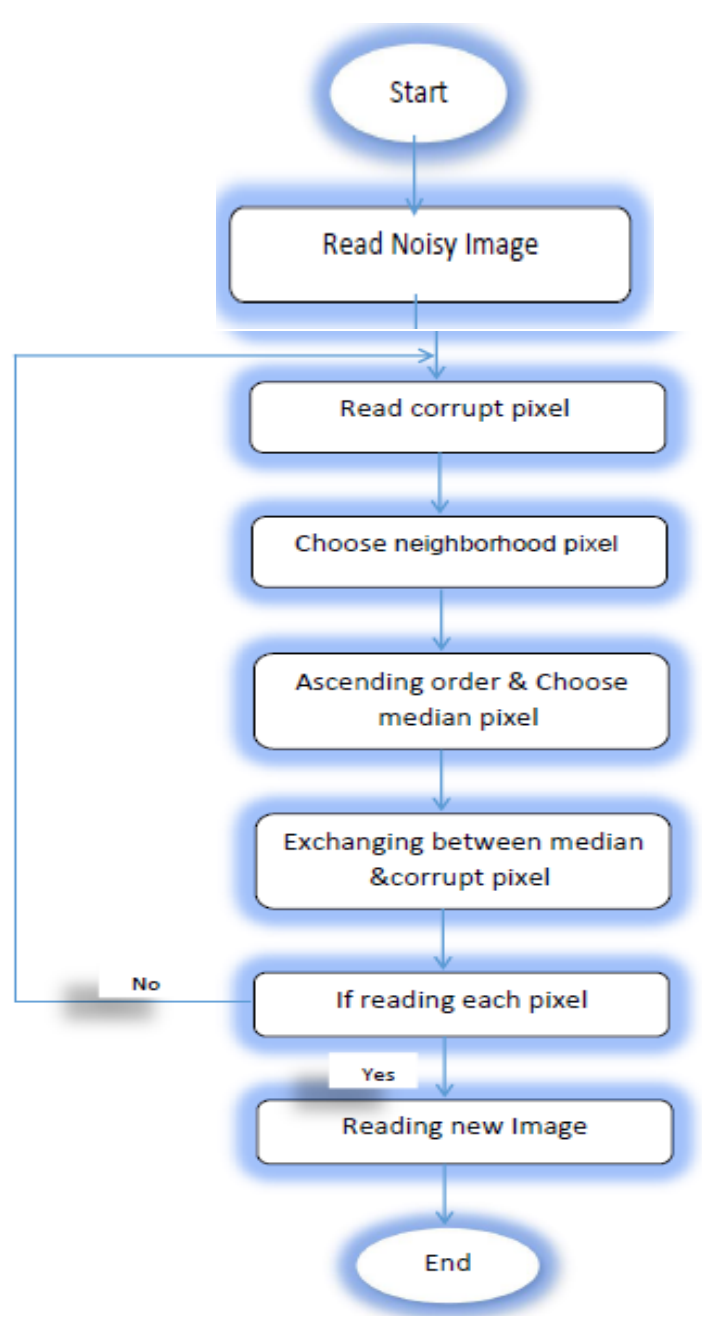

Fig.4.1 Flow chart of median filter algorithm
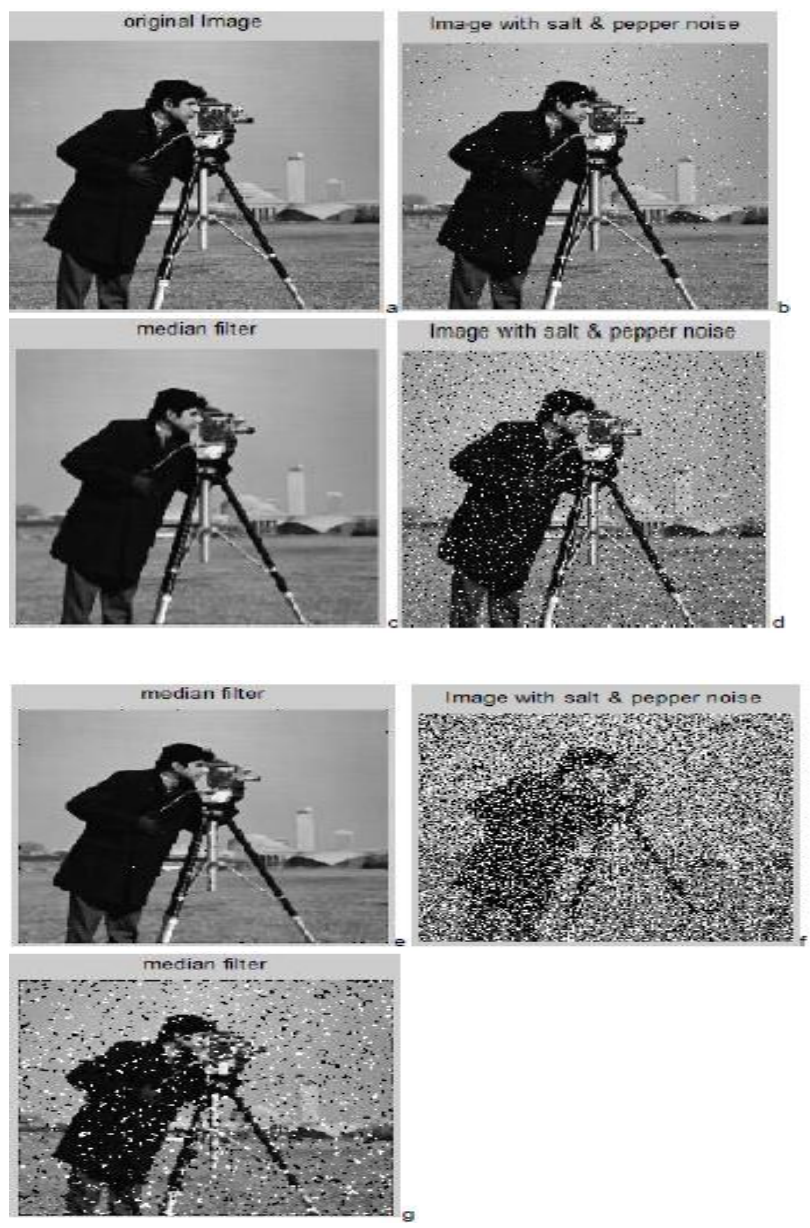

Fig.5.1 Comparing between the ratio of noise 'salt \& pepper' with median (3x3) filter cameraman. If a) Original image. b) Image with noise (0.01). And c) Image after median filter. d) Image with noise (0.1). And e) Image after median filter. f) Image with noise (0.5). And g) Image after median filter

\section{SimULATION \&RESULTS}

In this section we will use matlab program to obtain the characteristics of median filter. And will compare it with other filter. We will use the equation (3_3) for RMSe. The more active basic spatial filtering technique (algorithms) is (Median filter, Gaussian filter, Crimmins Speckle and Mean filter). It is important to eliminate the noise in the pictures before the some treatment later. The Median filter will help us by clean the black points and its name "Pepper".

\section{A. Grey scale images}

\section{a) Comparing between the ratio of noise 'salt \& pepper'} with median $(3 \times 3)$ filter

Example: the Image wills work on it, it's 'cameraman. If' shown in the figure $\left(5 \_1\right)$. And the result in table 1
TABLE1 COMPARING BETWEEN THE RATIO OF NOISE 'SALT \& PEPPER' WITH MEDIAN (3X3) FILTER

\begin{tabular}{|l|l|}
\hline Image with Ratio of noise & RMSe \\
\hline Image with noise (0.01). & 11.2108 \\
\hline Image with noise (0.1). & 12.9318 \\
\hline Image with noise (0.5). & 49.1466 \\
\hline
\end{tabular}

b) Comparing between the ratio of noise 'Gaussian' with median(3x3) filter:

Example: The Image wills work on it, it's ' pout. If ' shown in the figure (5_2). And the result in table 2 

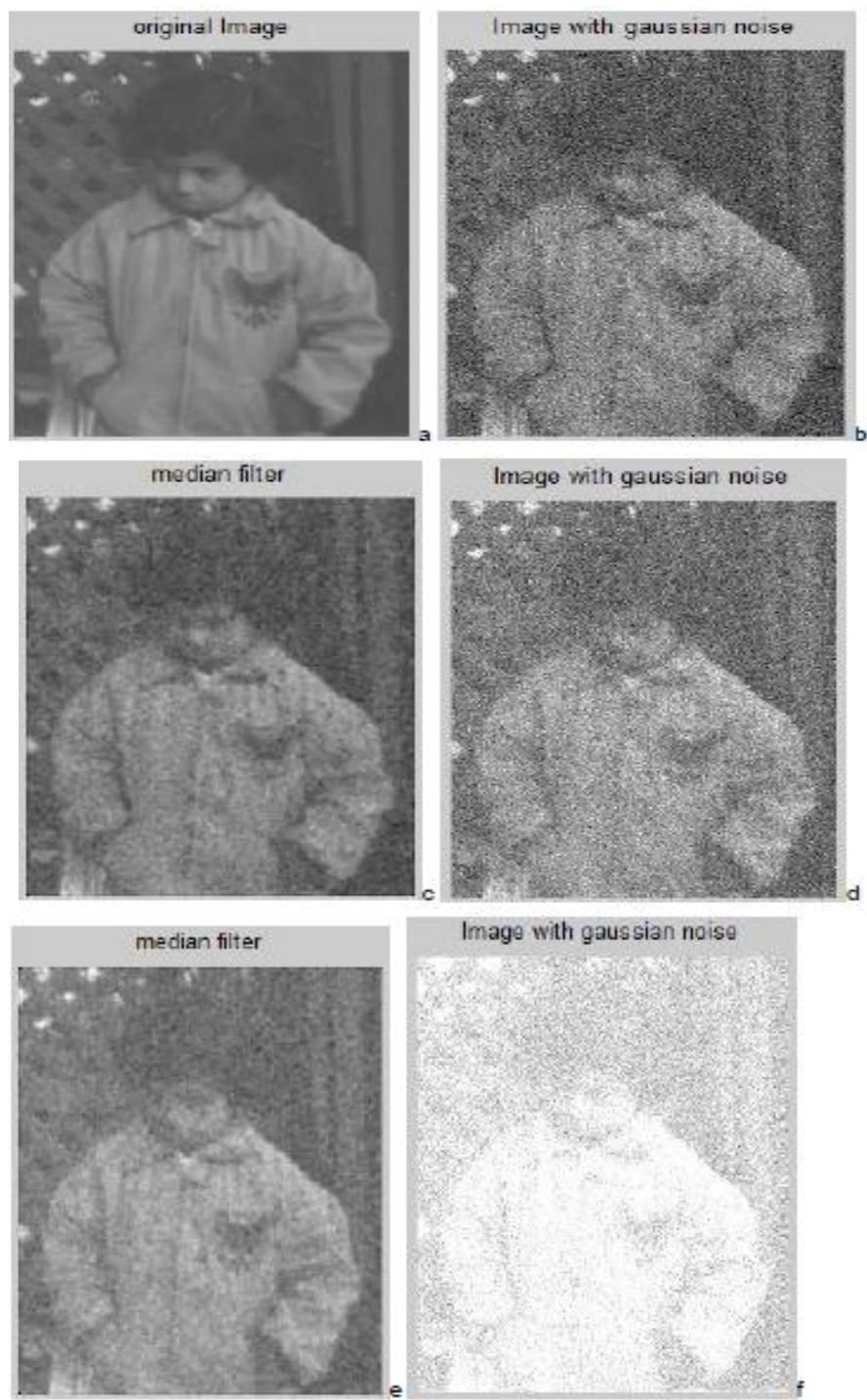

Image with gaussian noise

median filter

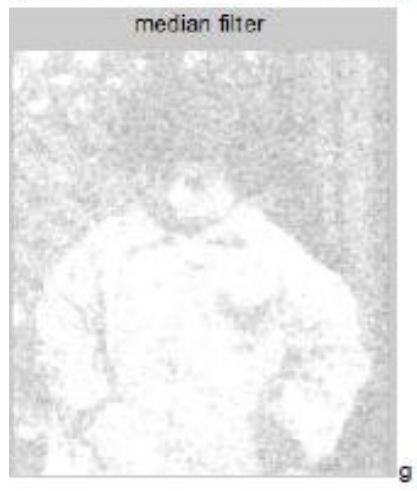

Fig. 5_2 Comparing between the ratio of noise 'Gaussian' with median( $3 \times 3)$ filter. Pout. If a) Original image. b) Image with noise

(0.01). And c) Image after median filter. d) Image with noise

(0.1). And e) Image after median filter. f) Image with noise (0.5). And g) Image after median filter.
TABLE 2 COMPARING BETWEEN THE RATIO OF NOISE 'GAUSSIAN' WITH MEDIAN(3X3) FILTER

\begin{tabular}{|l|l|}
\hline Image with Ratio of noise & RMSe \\
\hline Image with noise (0.01). & 11.2287 \\
\hline Image with noise (0.1). & 27.5483 \\
\hline Image with noise (0.5). & 123.6429 \\
\hline
\end{tabular}

c) Comparing between median( $3 \times 3)$ filter, average filter with noise ratio $(0.1)$ on the type of noise 'salt \& pepper':

Example: The Image wills work on it, it's 'trees. If' shown in the figure (5_3). And the result in table 3
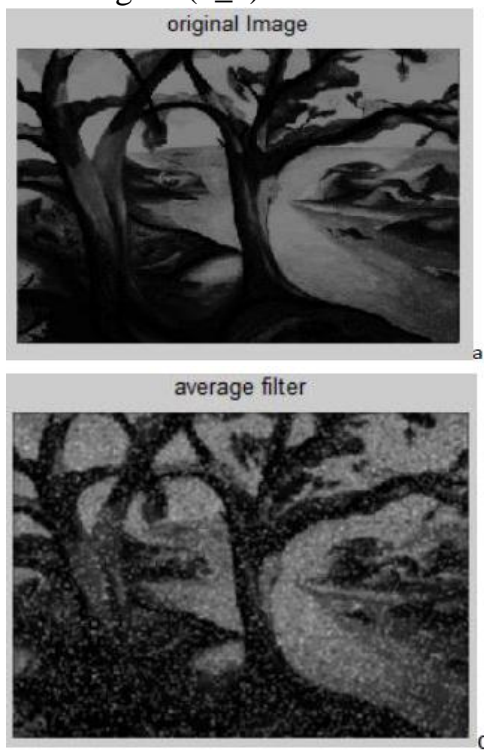

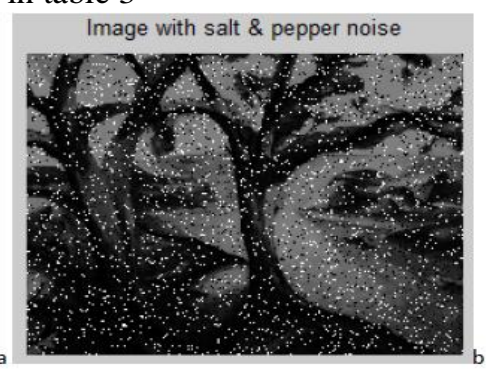

median filter

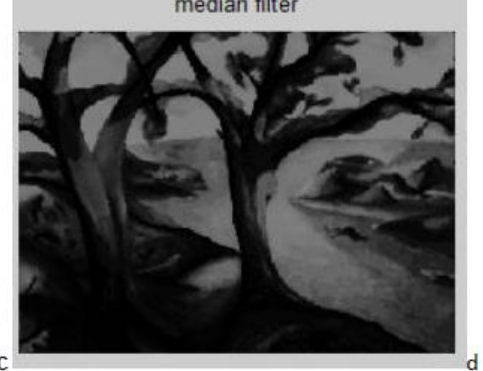

Fig. 5_3 Comparing between median ( $3 \times 3)$ filter, average filter with noise ratio $(0.1)$ on the type of noise 'salt $\&$ pepper' trees. If a) original image. b) Image with noise. c) Image after average filter. d) Image after median filter

TABLE 3 COMPARING BETWEEN MEDIAN(3X3) FILTER, AVERAGE FILTER WITH NOISE RATIO (0.1) ON THE TYPE OF NOISE 'SALT \& PEPPER'

\begin{tabular}{|l|l|}
\hline Type of filter & RMSe \\
\hline Average filter & $\mathbf{2 0 . 4 4 7 3}$ \\
\hline Median filter & $\mathbf{7 . 0 9 5 8}$ \\
\hline
\end{tabular}

d) Comparing between median ( $3 \times 3$ ) filter, average filter with noise ratio ( 0.1$)$ on the type of noise 'Gaussian':

Example: The Image wills work on it, it's ' moon. If ' shown in the figure (5_4). And the result in table 4 

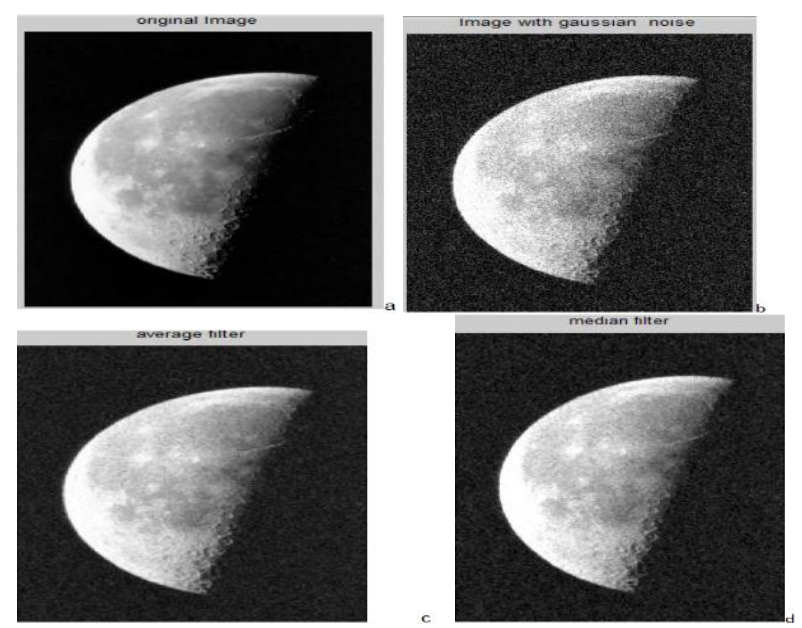
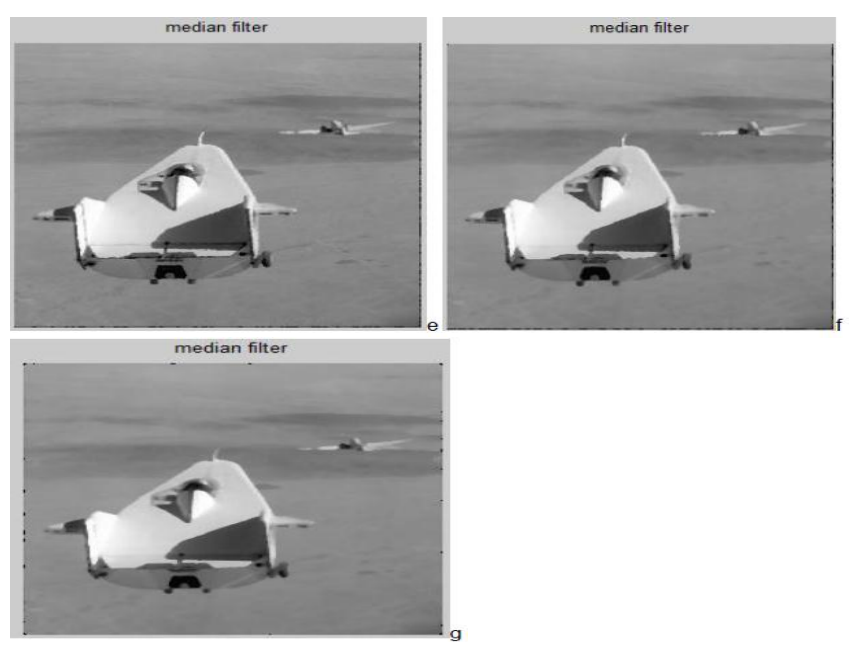

Fig.5_4 Comparing between median ( $3 \times 3)$ filter, average filter with noise ratio (0.1) on the type of noise 'Gaussian' moon. If a) original image. b) Image with noise. c) Image after average filter. d) Image after median filter.

Table 4 Comparing between median (3x3) filter, average filter with noise ratio $(0.1)$ on the type of noise 'Gaussian'

\begin{tabular}{|l|l|}
\hline Type of filter & RMSe \\
\hline Average filter & $\mathbf{2 7 . 3 0 6 3}$ \\
\hline Median filter & $\mathbf{2 7 . 2 6 4 4}$ \\
\hline
\end{tabular}

Fig. 5.5 Co (0.1) on th with noise. Image after median (5x5) filter. f) Image after median (6x6) filter. g) Image after median (7x7) filter.

\section{e) Comparing on median filter}

$(3 \times 3),(4 \times 4),(5 \times 5),(6 \times 6)$ and $(7 \times 7)$ with noise ratio (0.1) on the type of noise salt \& pepper:

Example: The Image wills work on it, it's 'liftingbody.png' shown in the figure (3_5). And the result in table 5

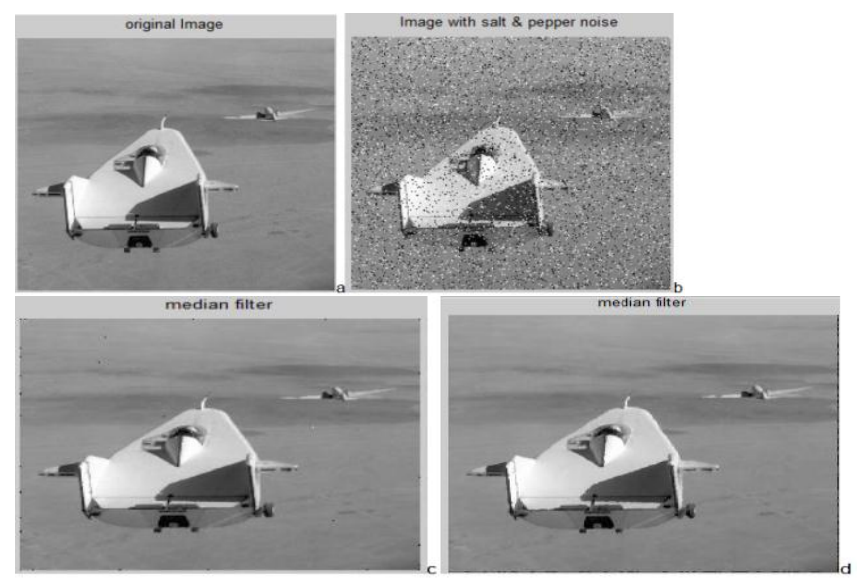

\begin{tabular}{|c|c|}
\hline Median( $3 \times 3)$ & 4.2357 \\
\hline Median $(4 \times 4)$ & 8.4191 \\
\hline Median $(5 \times 5)$ & 5.5990 \\
\hline Median $(6 \times 6)$ & 9.6263 \\
\hline Median $(7 \times 7)$ & 7.1866 \\
\hline
\end{tabular}

\section{V-II Color Scale images:}

a) Comparing between the ratio of noise 'salt \& pepper' with median $(3 \times 3)$ filter:

Example: the Image wills work on it, it's 'autumn. If' shown in the figure (5_6). And the result in table 6
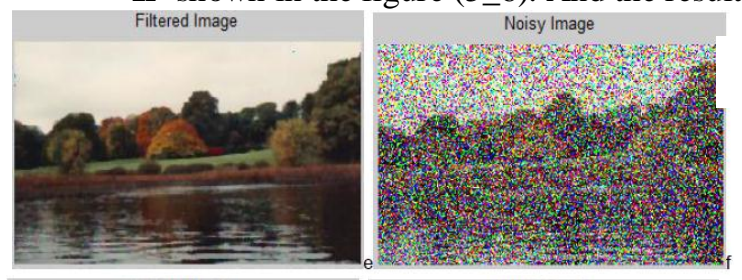

a

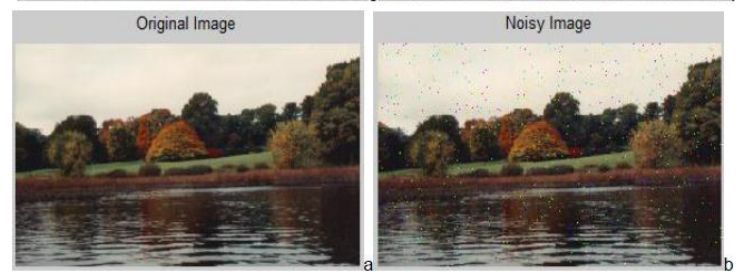




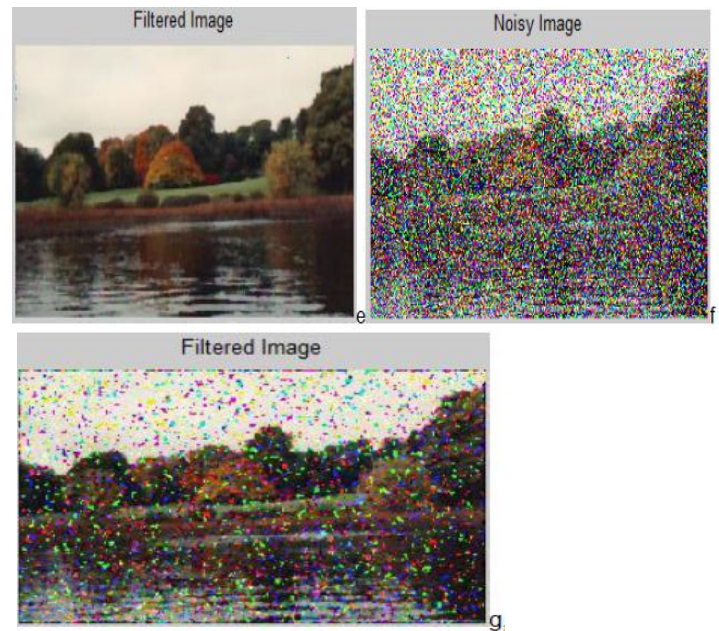

Table 6 Comparing between the ratio of noise 'salt $\&$ pepper' with $\operatorname{median}(3 \times 3)$ filter

\begin{tabular}{|l|l|}
\hline Image with Ratio of noise & RMSe \\
\hline Image with noise $(0.01)$. & 10.2513 \\
\hline Image with noise $(0.1)$. & 11.7994 \\
\hline Image with noise (0.5). & 51.2317 \\
\hline
\end{tabular}

b) Comparing between the ratios of noise 'Gaussian' with median $(3 \times 3)$ filter:

Example: the Image wills work on it, it's 'mohamed.jpg' shown in the figure (5_7). And the result in table 7

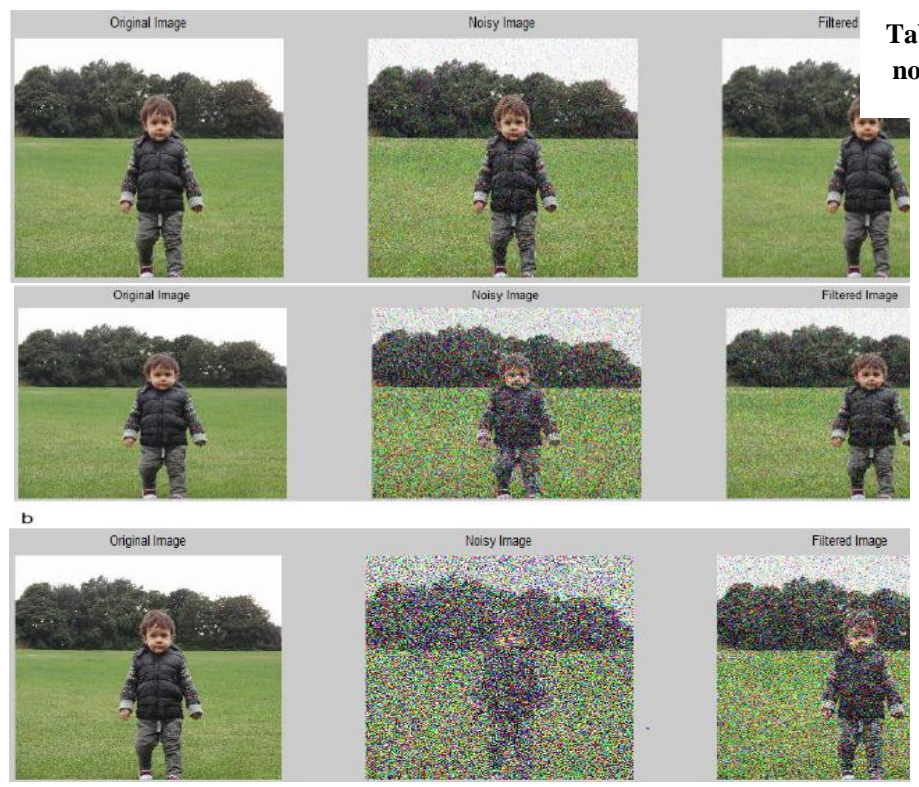

Fig. 5_7 Comparing between the ratios of noise 'Gaussian' with median (3x3) filter mohamed.jpg a) Image with ratio of noise $(0.01)$. b) Image with ratio of noise (0.1). c) Image with ratio of noise (0.5).

Table 7 Comparing between the ratios of noise 'Gaussian' with median (3x3) filter

\begin{tabular}{|l|l|}
\hline Image with Ratio of noise & RMSe \\
\hline Image with noise $(0.01)$. & 13.2875 \\
\hline Image with noise $(0.1)$. & 32.6123 \\
\hline Image with noise $(0.5)$. & 63.7626 \\
\hline
\end{tabular}

b) Comparing between median filter, average filter with noise ratio (0.1) on the type of noise 'salt \& pepper':

Example: the Image wills work on it, it's 'flwer.jpg' shown in the figure (5_8). And the result in table 8

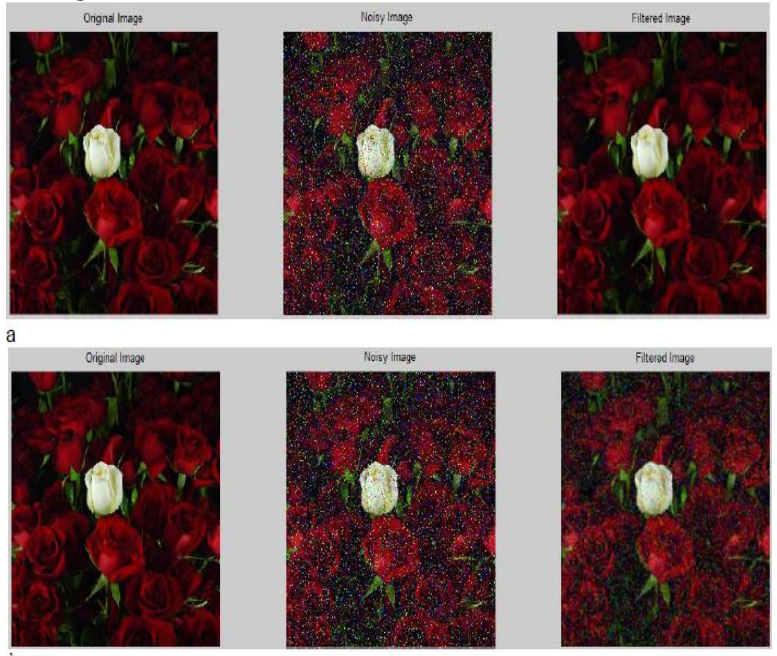

Fig. 5_8 Comparing between median filter, average filter with noise ratio (0.1) on the type of noise 'salt $\&$ pepper') flwer.jpg. Image with ratio of noise (0.1). a) Image filtered by median ( $3 \times 3)$ filter. b) Image filtered by average filter.

Table 8 Comparing between median filter, average filter with noise ratio (0.1) on the type of noise 'salt \& pepper')

\begin{tabular}{|l|l|}
\hline Type of filter & RMSe \\
\hline Median filter & 4.7323 \\
\hline Average filter & 20.9436 \\
\hline
\end{tabular}

c) Comparing between median filter, average filter with noise ratio (0.1) on the type of noise 'Gaussian': Example: the Image wills work on it, it's 'flwer2.jpg' shown in the figure (5_9). And the result in table 9

Fig. 5_10 compa noise ratio $(0.1)$ b) Image with $\mathrm{n}$ (4x4) filter e) Ir filter. g) Image a 
$\mathrm{b}$
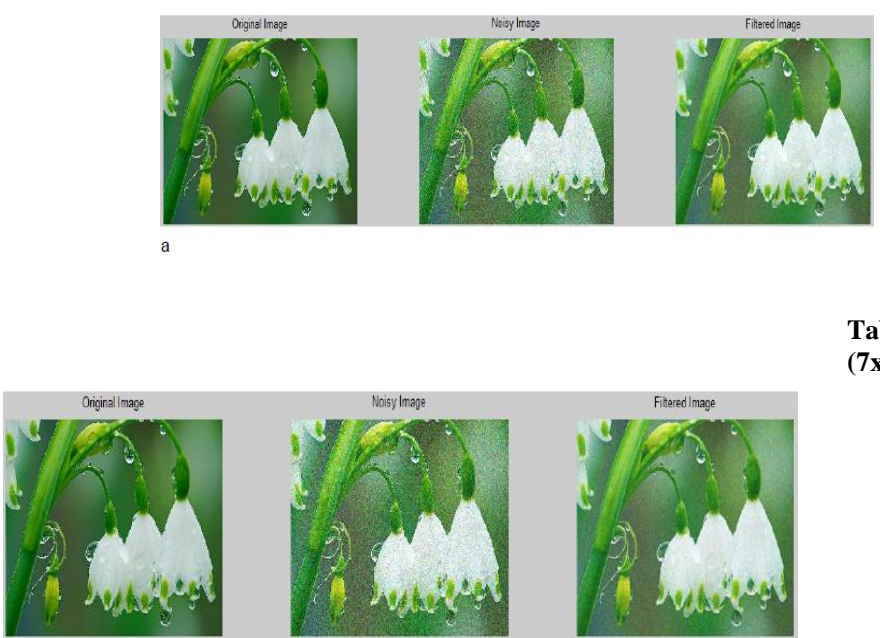

Fig. 5_9 Comparing between median filter, average filter with noise ratio (0.1) on the type of noise 'Gaussian' flwer2.jpg . Image with ratio of noise (0.1) 'Gaussian' a) Image filtered by median (3x3) filter. b) Image filtered by average filter.

Table 9 Comparing between median filter, average filter with noise ratio (0.1) on the type of noise 'Gaussian'

\begin{tabular}{|l|l|}
\hline Type of filter & RMSe \\
\hline Median filter & 28.2203 \\
\hline Average filter & 27.5852 \\
\hline
\end{tabular}

e) Comparing on median filter $(3 \times 3),(4 \times 4),(5 \times 5),(6 \times 6)$ and $(7 \times 7)$ with noise ratio $(0.1)$ on the type of noise salt $\&$ pepper:

Example: the Image wills work on it, it's 'flwer3.jpg' shown in the figure (5_10). And the result in table 10.
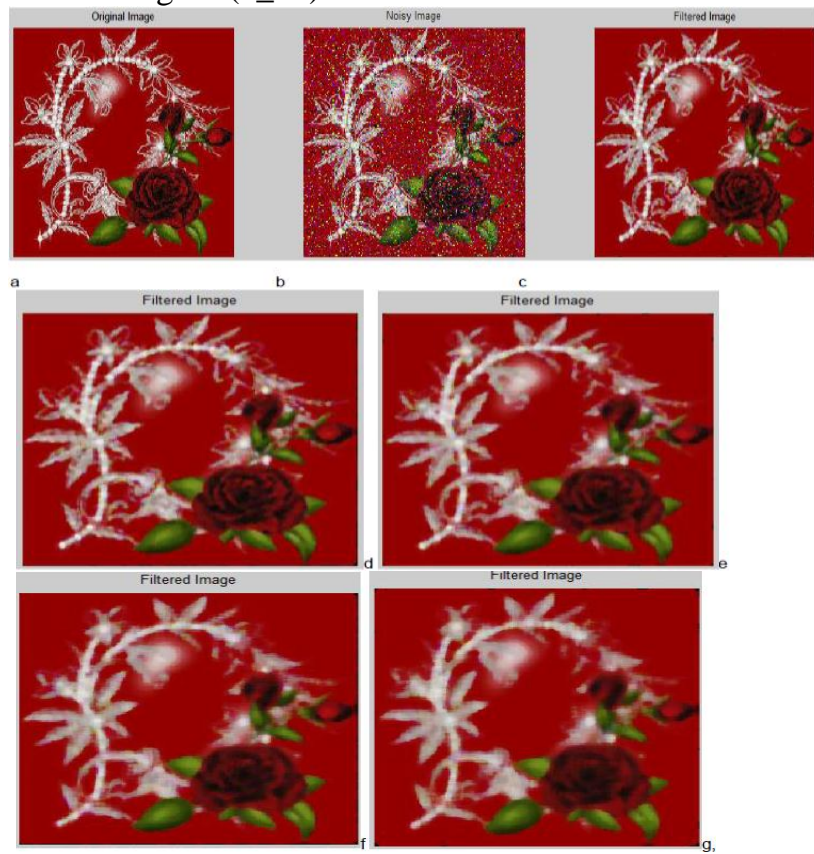

e
Table 10 Comparing on median filter $(3 \times 3),(4 \times 4),(5 \times 5),(6 \times 6)$ and $(7 \times 7)$ with noise ratio $(0.1)$ on the type of noise salt $\&$ pepper

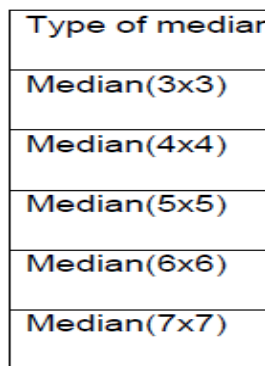

f) Comparing on median ( $3 \times 3)$ filter with noise ratio (0.5) on the type of noise salt \& pepper, to show the improving on repeat filter:

Example: the Image wills work on it, it's 'girle.jpg' shown in the figure (5_11). And the result in table 11.
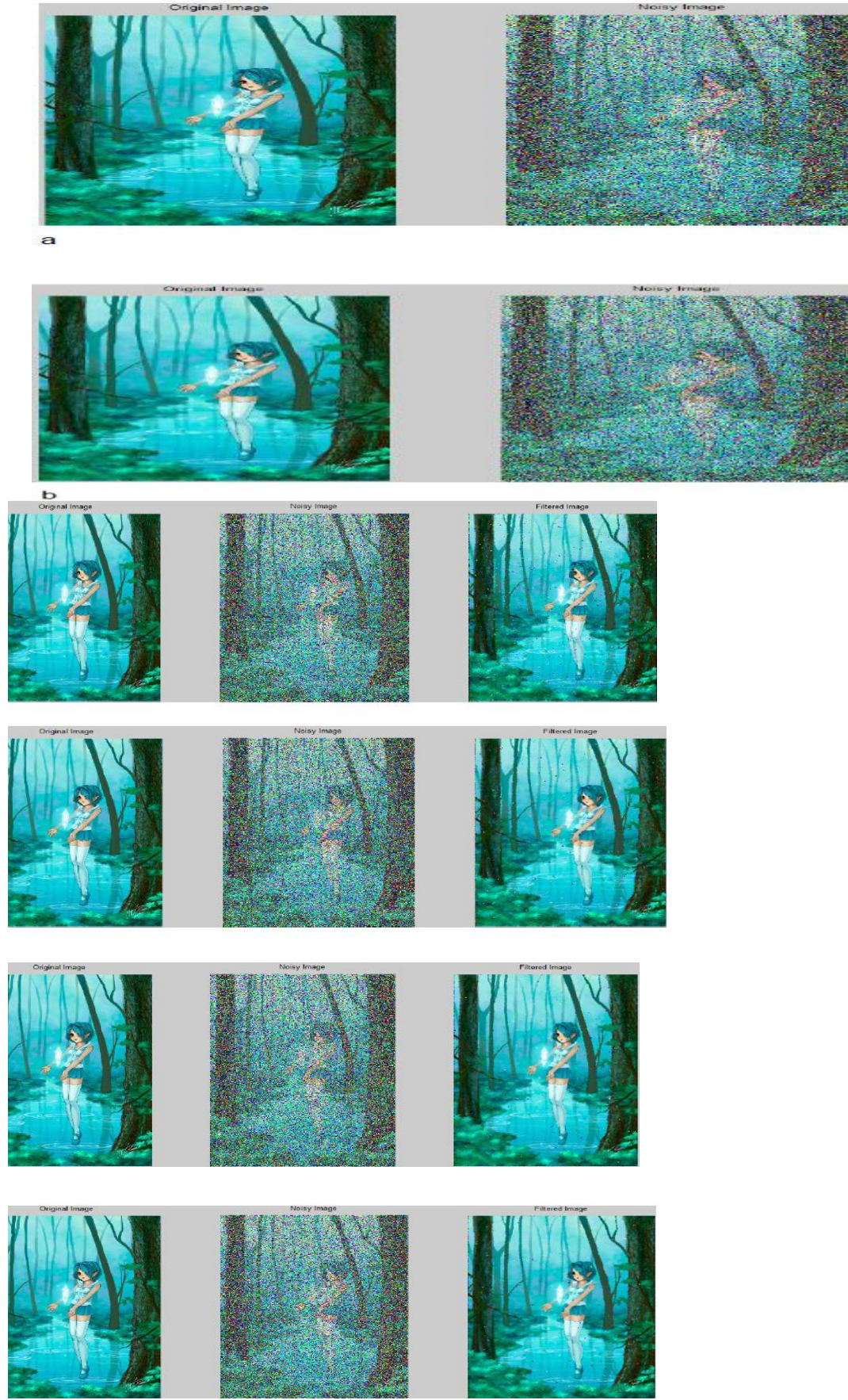

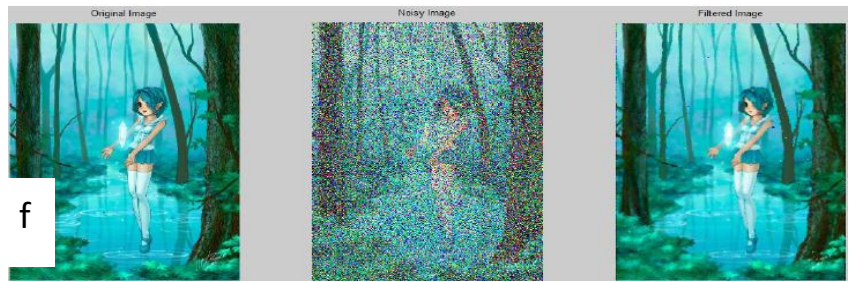

Fig.5_11 Comparing on median $(3 \times 3)$ filter with noise ratio $(0.5)$ on the type of noise salt $\&$ pepper, to show the improving on repeat filter 1girle.jpg a) first filter. b) Second filter. c) Third filter. d) Fourth filter. e) Fifth filter. f) Sixth filter. g) For more than 500 filter on the noisy Image.

Table 11 Comparing on median $(3 \times 3)$ filter with noise ratio (0.5) on the type of noise salt \& pepper, to show the improving on reneat filter

\begin{tabular}{|c|c|}
\hline No. of median filter & RMSe \\
\hline Median(3×3)_1 & 48.6521 \\
\hline Median(3×3)_2 & 28.4349 \\
\hline Median(3×3)_3 & 20.2626 \\
\hline Median(3×3)_4 & 16.8983 \\
\hline Median(3×3)_5 & 15.3469 \\
\hline Median(3×3)_6 & 14.8903 \\
\hline Median(3x3)_for more than 10 & 14.3785 \\
\hline $\begin{array}{l}\text { Median }(3 \times 3) \_ \text {for very greater } \\
\text { More than } 500\end{array}$ & 14.3540 \\
\hline
\end{tabular}

g) Comparing between (salt \& pepper and Gaussian) noise:

Example: the Image wills work on it, it's 'lenna.jpg' with median (3x3) filter, average filter as shown in the figure (3_12). The result of RMSe is in table 12, the ratio of noise (0.1).
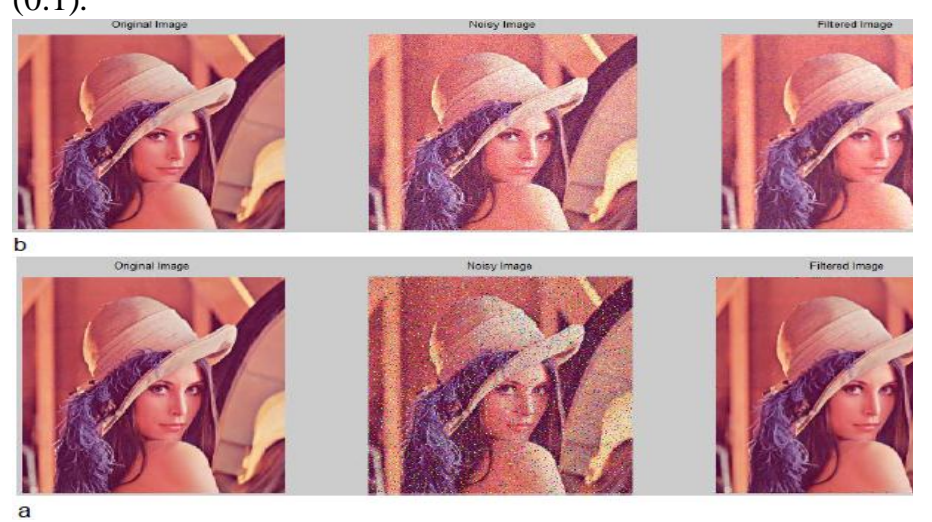
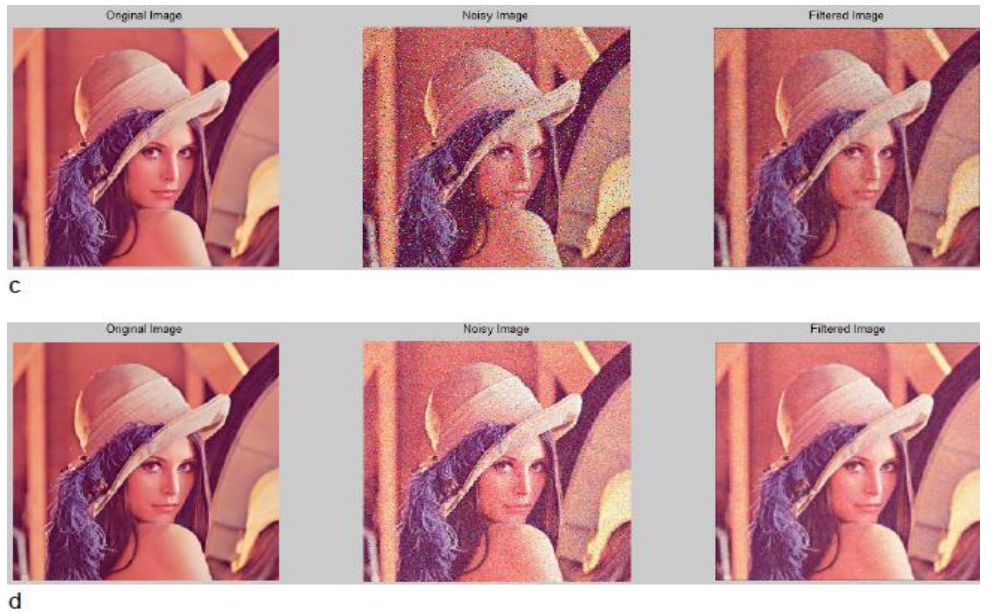

Fig.3_12 Comparing between (salt $\&$ pepper and Gaussian) noise lenna.jpg a) Median filter with salt $\&$ pepper noise. b) Median filter with Gaussian noise. c) Average filter with salt \& pepper noise. d) Average filter with Gaussian noise

Table 12 Comparing between (salt $\&$ pepper and Gaussian)

\begin{tabular}{|l|l|l|}
\hline Type of filter & Salt \& Pepper noise & Gaussian noise \\
\hline Median filter & 5.3957 & 27.4331 \\
\hline Average filter & 17.1501 & 26.2788 \\
\hline
\end{tabular}

\section{Conclusions}

As known in this paper the tables from table 1 to table 6 are illustrated the results including the gray scale images and also tables from table 7 to table 12 are including weather the situation is for color scale images.

So, we will the surmise such as comparing between for each tow both similarity cases. Since, from table1 for Gray image we can see when noise ratio is increase the RMSe is also increase, with salt \& pepper noise. And also for color image the table 6, when noise increase the RMSe also increase for (salt \& pepper noise).

Also looking for table 2 we can see when noise ratio is increase the RMSe is also increase, with Gaussian noise, for Gray image. And in the same time for color image in table 7, when noise increases the RMSe also increase for (Gaussian noise).

Therefore, that can say, in applying median filter with their condition here, they tend to remove many of the features of the pictures or keep a lot of noise boost when the noise is increase.

Looking at table 3 for Gray image can see the median filter is better than Average filter to remove the salt \& pepper noise. Whatever, for table 8 we can see the median filter is better 
than Average filter to remove the salt \& pepper noise in color image.

So that for table 4 the median filter seem better than order filter to remove Gaussian against the average filter in Gray image. But in table 9 the median filter appears not better than average filter for color image.

For this reason can say later median filter is good to remove salt and pepper noise rather than other type of noise, and is also maintain the edge of picture.

Clearly also, table 5 shown us the median $(3 \times 3)$ is the better, that because the median $(3 \times 3)$ is nearer to the corrupt pixel for Gray image. But the table 10 into seem the median $(3 \times 3)$ is the better, that because the median $(3 \times 3)$ is nearer to the corrupt pixel in color image. So that to remove noise from image with a good form must be chosen median ( $3 \times 3)$ filter) and we see when the array of median is even number it will be like mean filter.

In table 11 can note when we repeat the median filter on the noisy image to enhancement it, the RMSe will reduce.

Table 12 has illustrated us, for another example, that the median filter is better from average filter to remove salt \& pepper noise and saving the edge of image. So that when the noise is Gaussian, the median filter is less good from average filter, for color image and noise ratio (0.1).

Lastly the Median filtering is better than mean (average) filter because the median filter when be median $(3 \times 3)$ or odd number will replace the corrupt pixel by the median pixel while the mean filter like median with even number will take the average value to replace the corrupt, so median filter will maintain the feature of image

\section{References}

[1] M. Rouse,http://whatis.techtarget.com/definition/fuzzy-logic,whats.com, July 2006

[2] P. S. a. P. B. Roli Bansal,(2007) "A Simplified Fuzzy Filter for Impulse Noise," in WCECS 2007, San Francisco, USA, 2007.

[3] J.-H. W. A. H.-C. CHIU, (1999)"HAF: an Adaptive Fuzzy Filter for Restoring Highly," in Proc. Natl. Sci. Counc. ROC(A), Keelung, Taiwan, R.O.C., Department of Electrical Engineering, (Received November 19, 1998; Accepted March 11, 1999), pp. No. 5, 1999. pp. 630-643.

[4] K. Bodduna and I. I. O. S. E. \&, (2013)"A Novel Random-valuedimpulse Noise Detection and," IEEE, 07 Jan 2013. [Online]. Available: http://www.srajesh.in/papers/21.pdf. [Accessed 42 2015].

[5] S. B. Saravanakumar.S, "Impulse Noise Removal Using Optimal Direction Method and Adaptive Switching Median Filter, "Anna University of Technology, Coimbatore, India.

[6] J. P. Steiner, A. Dobbins and E. J. Blair, (2003)"Root properties and يناير 29 2003. [Online]. Available: http://ieeexplore.ieee.org/xpl/artic

leDetails.jsp?tp $=\&$ arnumber $=1164543 \&$ queryText $\% 3$ DYou + can

+repeat+the+median+filter+on+the+image . [Accessed 202 2015].

[7] S. E. Baugh,(2008) Computer Vision and Image Processing a Practical Approach using CVIP Tools, Prentice Hall PTR, 2008.

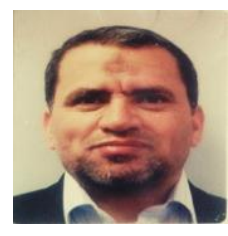

Dr. Yahya Ali Lafta was born in Hilla, Iraq, in February 1960. He graduated in engineering Electronics aviation in 1982

From academic engineering air Force Yugoslavia and received the M.Sc. degree in 1984, both from the University of Belgrade, Yugoslavia In 2012, he received the Ph.D. degree from the University of LJMU, U.K.

$\mathrm{He}$ is currently a Lecturer at the Department electronics\& communication faculty engineering of Kufa, Iraq. His research interests include video coding and networking, compressed-domain algorithms, robust coding techniques for wireless communications, and multimedia networks.

ID: IAE0317007 